\title{
Eosinophilic myositis resulted from Sarcocystis infection in prime marbled beef of J apanese black cattle
}

\author{
Tohru Kimura \\ Center for Experimental Animals, \\ National Institutes of Natural Sciences, \\ 38, Nishigo-naka, Myodaiji, Okazaki, Aichi, 444-8585, Japan \\ Tel. 0564-55-7882, FAX: 0564-55-7881, E-mail: kimura@nips.ac.jp \\ Received:11-06-2011, Accepted: 19-06-2011, Published Online: 22-09-2011 \\ doi: $10.5455 /$ vetworld.2011.500-502
}

\begin{abstract}
Partial changes of color (greenish to brownish) were found in prime marbled beef of Japanese black cattle. The disseminated lesions of the skeletal muscles were histopathologically examined in relation to Sarcocystis infection. The lesions in the muscles showed granulomas with inflammatory cell infiltration. The sarcocysts had a distinct wall, which was radically striated by palisading villar protrusions. The sarcocyst wall was surrounded by degenerative eosinophils and necrotic muscle fibers. In conclusion, eosinophilic myositis in prime marbled beef of Japanese black cattle resulted from Sarcocystis spp. infection. The muscular lesions were characterized by the presence of granulomas and capsulated sarcocysts surrounded by numerous eosinophils.
\end{abstract}

Key w ords: eosinophilic myositis, granulomas, Japanese black cattle, sarcocyst, Sarcocystis spp.

\section{I ntroduction}

Previous studies suggested that Sarcocystis spp., often found in association with granulomatous myositis, should be responsible for eosinophilic myositis (Jensen et al., 1986; Gajadhar et al., 1986; Gajadhar and Marquardt, 1991; Wouda et al., 2006). Eosinophilic myositis is a relatively rare disease in cattle observed in meat inspection after slaughter. Most investigators describe a high prevalence of Sarcocystis infection in heart, esophagus, diaphragm and tongue of slaughtered cattle. Although the skeletal muscles are the most often eaten parts of beef, the prevalence of Sarcocystis is relatively low in these muscles. In addition, skeletal muscles are not severely affected by Sarcocystis.

The prevalence of Sarcocystis spp. cysts was lower in Japanese beef $(6.31 \%)$ than in beef imported from America (36.78\%) or Australia (29.49\%) (Ono and Ohisumi, 1999). Marbled beef of Japanese black cattle is highly valued as brand-name beef produced in the specified farms.

I found a partial change of color in prime beef of Japanese black cattle. In this study, the disseminated lesions of the skeletal muscles were histopathologically examined in relation to Sarcocystis infection.

\section{Materials and Methods}

Samples of muscles from the hindquarters in
Japanese black cattle were excised in the slaughter house in Matsuzaka-city and submitted to our institutes. The specimens containing visible lesions were fixed in $10 \%$ neutral buffered formalin, processed by routine methods and embedded in paraffin wax. 4- $\mu$ m paraffin sections were stained with hematoxylin and eosine (HE) and by van Gieson's and Weigert's staining.

\section{Results}

Macroscopic findings: The marbled beef had welldemarcated, oval or elongated greenish or grayishgreen lesions up to $2-10 \mathrm{~mm}$ in length and 2-8 $\mathrm{mm}$ in diameter. The small disseminated lesions were a typical form of eosinophilic myositis which resulted in carcass condemnation (Figure I). The large-sized lesions were discrete focal stripes or patches. In some portions, there was a rigid bulging of brownish lesions (Figure II).

Histopathological findings: The lesions in the marbled muscles showed granulomas with inflammatory cell infiltration. The granulomatous lesions had a central core consisting of degenerative eosinophilic leucocytes and remnants of necrotic skeletal muscle fibers. Among the marbled muscle fibers, there was accumulation of mixed inflammatory cells, mainly eosinophils with some neutrophils, macrophages and plasma cells, as well as erythrocytes 


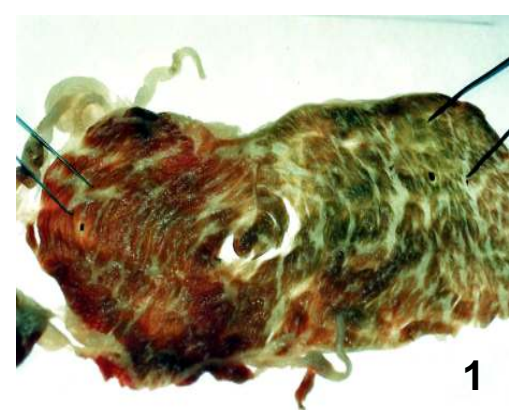

1

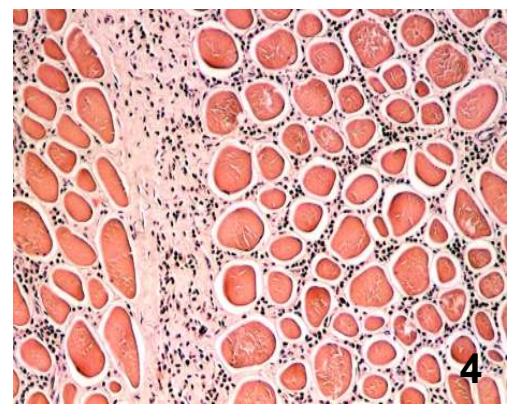

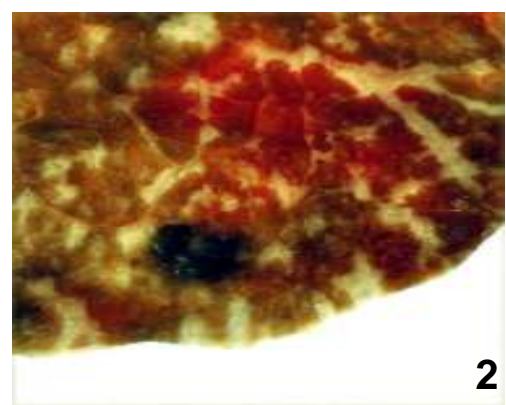

2

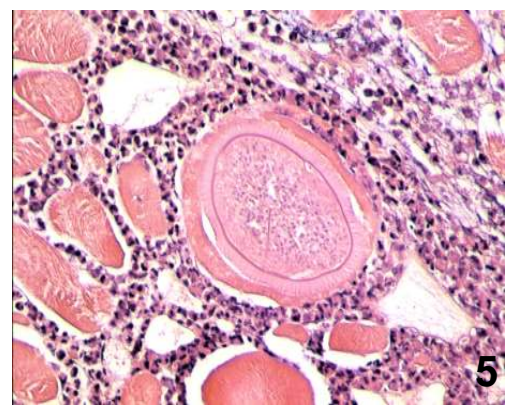

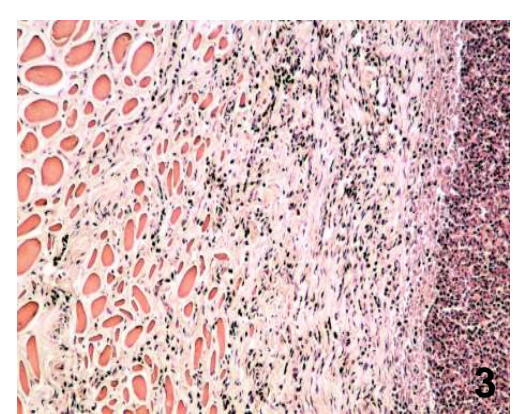

Figure-I. Macroscopic photograph. Oval or elongated brownish lesions are seen in the marbled beef.

Figure-II. Macroscopic photograph. A discrete Figure-II. Macroscopic photog
large-sized lesion in the muscle.

Figure-III. Microscopic photograph. Granuloma with inflammatory cell infiltration is seen. $\times 100$, HE stain.

Figure-IV. Microscopic photograph. Diffuse eosinophil infiltration seen in the connective tissue of normal skeletal muscles. $\times 150, \mathrm{HE}$ stain.

Figure-V. Microscopic photograph. The capsulated sarcocyst is surrounded by degenerative eosinophils and necrotic muscle fibers. $\times 150$, HE stain.

(Figure III). Pale to neutral staining eosinophils were observed around the sarcocysts with thick cyst wall. There was also diffuse eosinophil infiltration in the connective tissue of normal skeletal muscles (Figure IV).

The sarcocysts had a distinct wall, which was radically striated by palisading villar protrusions. The sarcocyst wall was surrounded by degenerative eosinophils and necrotic muscle fibers (Figure IV). Adjacent to the trapped sarcocyst, this cyst were enclosed with a thick outer rim of degenerative hyalinized remnants of muscle fibers.

Histopathological examinations revealed that eosinophilic miositis were closely associated with Sarcocystis infection.

\section{Discussion}

Muscle sarcocystosis is a parasitic infection acquired by ingestion of sarcocysts of Sarcocystis spp. in feces from an infected carnivore. Sarcocystis spp. have 2-host life cycle between carnivores and herbivores. In this life cycle, cattle are known to serve as the intermediate host. In the previous study, the cows experimentally challenged with sporocysts showed various clinical signs with myositis (Savini et al., 1996). Histopathologically, skeletal myositis was associated with a mononuclear cell infiltration (primarily lymphocytes). Degenerating sarcocysts in muscles elicited a mononuclear cell (mainly lymphocytic) response as early as 1 to 3 months after infection.
In the present study, histopathological examinations indicated that granuloma-associated sarcocysts were partially destroyed or enclosed by dense, organized accumulations of inflammatory cells. These results showed that the thick cyst walls in the lesions were identical to those of Sarcocystis hirsute. The thick walled sarcocyst cannot be distinguished whether it is Sarcocystis hirsute or Sarcocystis hominis. However, Sarcocystis hominis has not yet been reported in Japan.

The pathogenesis of eosinophilic myositis is not clear and this muscular lesion is not always found in cattle suffering from Sarcocystis spp. The incidence of eosinophilic myositis is usually low in cattle (Wouda et al., 2006). This breed of cattle was mainly fed with concentrated fed. It is uncertain whether the cattle made some contact with carnivores as the definitive host. The histopathological examinations showed 2 kinds of morphological changes as follows: granulomas with peripheral cell infiltration and capsulated sarcocysts surrounded by degenerative eosinophils. The initial cellular response seemed to be induced by substances (eosinophil chemoattractant) released from the sarcocysts during their normal metabolic process. These findings provided a presumption that cattle with eosinophilic myositis were predisposed to produce $\operatorname{IgE}$ in response to Sarcocystis bradyzoite antigen, and that eosinophilic myositis represented an abnormal response to sarcocyst degeneration, including a host-dependent, 
Sarcocystic, type-I hypersensitivity (Granstrom et al., 1989 and Granstrom et al., 1990). Mononuclear cell infiltration is predominant feature of myositis associated with early Sarcocystis infention in calves without lesions of eosinophilic myositis. It is probable that eosinophilic myositis develops around degenerating sarcoysts in the later stage.

In conclusion, eosinophilic myositis in prime marbled beef of Japanese black cattle resulted from Sarcocystis spp. infection. The muscular lesions contained granulomas and capsulated sarcocysts surrounded by numerous eosinophils.

\section{Acknowledgements}

I thank Ms. Mina Kimura and Mr. Gen Kimura for their expert technical assistance.

\section{References}

1. Gajadhar AA, Yates WDG, Allen JR. (1986). Association of eosinophilic myositis with an unusual species of Sarcocystis in a beef cow. Can J Vet Res 51:373-378.

2. Gajadhar AA, Marquardt WC. (1991). Ultrastructural and transmission evidence of Sarcocystis cruzi associated with eosinophilic myositis in cattle. Can J Vet Res 56: 41-46.

3. Granstrom DE, Ridley RK, Baoan Y, Gershwin LJ, Nesbitt PM, Wempe LA. (1989). Type-I hypersensitivity as a component of eosinophilic myositis (muscular sarcocystosis) in cattle. Am J Vet Res 50: 571-574.

4. Granstrom DE, Ridley RK, Baoan Y, Gershwin LJ, Briggs DJ. (1990). Immunofluorescent localization of Sarcocystis cruzi antigens, $\operatorname{IgG}$ and $\mathrm{IgE}$, in lesions of eosinophilic myositis in cattle. J Vet Diagn Invest 2: 147-149.

5. Jensen R, Alexander AF, Dahlgren RR, Jolley WR, Marquardt WC, Flack DE, Bennett BW, Cox MF, Harris CW, Hoffmann GA, Troutman RS, Hoff RL, Jones RL, Collins JK, Hamar DW, Cravans RL. (1986). Eosinophilic myositis and muscular sarcocytosis in the carcasses of slaughtered cattle and lambs. Am J Vet Res 47: 587-593.

6. Ono M, Ohsumi T. (1999). Prevalence of Sarcocystis spp. cysts in Japanese and imported beef (Loin: Musculus longissimus). Parasitology International 48: 91-94.

7. Savini G, Ducsmore JD, Robertson ID. (1996). Studies on pathogenesis, tissue infection and congenital transmission in cows experimentally infected with Sarcocystis cruzi by various routes. Vet Parasitol 64: 319-327.

8. Wouda W, Snoep JJ, Dubey JP. (2006). Eosinophilic myositis due to Sarcocystis hominis in a beef cow. J Comp Path 135: 249-253. 